\title{
Dust-grain processing in circumbinary discs around evolved binaries. The RV Tauri spectral twins RU Centauri and AC Herculis *
}

\author{
C. Gielen ${ }^{1}$, H. Van Winckel ${ }^{1}$, L. B. F. M. Waters ${ }^{1,2}$, M. Min ${ }^{2}$, and C. Dominik ${ }^{2}$
}

\author{
1 Instituut voor Sterrenkunde, Katholieke Universiteit Leuven, Celestijnenlaan 200D, 3001 Leuven, Belgium \\ e-mail: Clio.Gielen@ster. kuleuven. be \\ 2 Sterrenkundig Instituut Anton Pannekoek, Universiteit Amsterdam, Kruislaan 403, 1098 Amsterdam, The Netherlands \\ Received 20 July 2007 / Accepted 4 September 2007
}

\section{ABSTRACT}

\begin{abstract}
Context. We study the structure and evolution of the circumstellar discs around evolved binaries and their impact on the evolution of the central system.

Aims. By combining a wide range of observational data and techniques, we aim to study in detail the binary nature of RU Cen and $\mathrm{AC} \mathrm{Her,} \mathrm{as} \mathrm{well} \mathrm{as} \mathrm{the} \mathrm{structure} \mathrm{and} \mathrm{mineralogy} \mathrm{of} \mathrm{the} \mathrm{circumstellar} \mathrm{environment.}$

Methods. We combine a multi-wavelength observational program with a detailed 2D radiative transfer study. Our radial velocity program is instrumental in the study of the nature of the central stars, while our Spitzer spectra complimented with the broadband spectral energy distribution (SED) are used to constrain mineralogy, grain sizes and physical structure of the circumstellar environment.

Results. We determine the orbital elements of RU Cen showing that the orbit is highly eccentric with a large velocity amplitude despite the rather long period of 1500 days. The infrared spectra of both objects are very similar and the spectral dust features are dominated by magnesium-rich crystalline silicates. The small peak-to-continuum ratios are interpreted as being due to large grains. Our model contains two components with a cold midplain dominated by large grains, and the near- and mid-IR which is dominated by the emission of smaller silicates. The infrared excess is well modelled assuming a hydrostatic passive irradiated disc. The profilefitting of the dust resonances shows that the grains must be very irregular.

Conclusions. These two prototypical RV Tauri pulsators with circumstellar dust are binaries where the dust is trapped in a stable disc. The mineralogy and grain sizes show that the dust is highly processed, both in crystallinity and grain size. The cool crystals show that either radial mixing is very efficient and/or that the thermal history at grain formation has been very different from that in outflows. The physical processes governing the structure of these discs are very similar to those observed in protoplanetary discs around young stellar objects.
\end{abstract}

Key words. stars: AGB and post-AGB - stars: evolution - stars: binaries: general - stars: circumstellar matter stars: individual: RU Cen - stars: individual: AC Her

\section{Introduction}

The RV Tauri class of objects contains highly luminous stars showing large-amplitude photometric variations with alternating deep and shallow minima. The members are located in the high luminosity end of the population II instability strip and the photometric variations are interpreted as being due to radial pulsations. There are two different photometric classes: the RVa stars are objects with a constant mean magnitude while the RVb objects display a long-term variation in their mean magnitude. Preston et al. (1963) introduced a spectroscopic classification of the RV Tauri stars, using unfortunately the same alphabetic letters for the naming: RVA objects show strong absorption lines, RVB objects are of a somewhat hotter spectral type but are weak lined with enhanced $\mathrm{CN}$ and $\mathrm{CH}$ bands. The RVC objects are also weak lined but show no enhanced $\mathrm{CN}$ and $\mathrm{CH}$ molecular band strength. A significant fraction of the RV Tauri stars show

* Based on observations obtained at the European Southern Observatory (ESO), La Silla, observing program 072.D-0263, on observations made with the $1.2 \mathrm{~m}$ Flemish Mercator telescope at Roque de los Muchachos, Spain, the $1.2 \mathrm{~m}$ Swiss Euler telescope at La Silla, Chile and on observations made with the Spitzer Space Telescope (program id 3274), which is operated by the Jet Propulsion Laboratory, California Institute of Technology under a contract with NASA. a large IR excess due to circumstellar dust and Jura (1986) identified them as post-AGB objects on the basis of this IR excess, their luminosities and mass-loss history.

The photospheric content of RV Tauri stars is, however, very different from what could be expected in post-3rd dredge-up objects: they do not show high C-abundances or s-process overabundances but instead often show a depletion pattern in their photospheres (Giridhar et al. 1994, 1998, 2000; Gonzalez et al. 1997a,b; Van Winckel et al. 1998; Maas et al. 2005). This abundance pattern is the result of gas-dust separation followed by reaccretion of the gas, which is poor in refractory elements. Waters et al. (1992) proposed that the most likely circumstance for this process to occur is when the dust is trapped in a circumstellar disc. This depletion phenomenon is also observed in binary post-AGB stars with a disc (Van Winckel et al. 1995). This led Van Winckel et al. (1999) to suggest that the depleted RV Tauri stars must also be binaries with a disc. The likely presence of a Keplerian circumstellar disc was further shown by the systematic study by De Ruyter et al. (2005); De Ruyter et al. (2006) of a large sample of binary post-AGB and RV Tauri stars.

Blöcker (1995) list the post-AGB evolutionary tracks of single stars of different initial mass. Evolutionary tracks for binary post-AGB stars have not yet been determined, however, since we find evidence that these stars have been shortcut on their AGB 
evolution (Sect. 8), we expect longer lifetime scales because of the expected lower core masses. Typical post-AGB lifetimes of both stars are estimated to be of the order of $\sim 10^{4} \mathrm{yr}$.

To investigate the special evolutionary status of RV Tauri stars and to research in detail the interplay between the photospheric and circumstellar environment in these systems, we focus in this paper on two well known RV Tauri stars: AC Her and RU Cen.

AC Her has been extensively studied in the literature. It is a binary RV Tauri star of photometric class a, with an orbital period of 1200 days (Van Winckel et al. 1998). It is a very regular pulsator with a formal pulsation period (timespan between two successive deep photometric minima) of 75.5 days (Zsoldos 1993). The mean magnitude of ACHer is $m_{V}=7.69 \mathrm{mag}$ and the amplitude $\Delta m_{V}=2.31 \mathrm{mag}$ (Lloyd Evans 1985). The presence of two strong shock waves in every formal pulsation cycle, causing line-profile deformations in the spectra of AC Her and R Scuti has been discussed by Gillet et al. (1989, 1990). AC Her shows a chemical depletion pattern (Van Winckel et al. 1998; Giridhar et al. 2000) that is attributed to the presence of a stable Keplerian dusty disc. The presence of such a disc has also been proposed by Jura \& Kahane (1999) who interpret the detection of weak CO rotational emission lines with a small velocity width (Bujarrabal et al. 1988; Jura et al. 1995) as a signature of such a long-lived dust reservoir. The presence of highly crystalline silicates in the infrared spectrum (Molster et al. 1999) and the strong millimeter continuum flux from large dust grains (Shenton et al. 1995; Jura \& Kahane 1999) further corroborate this conclusion. Jura et al. (2000) claimed to have resolved the circumstellar material around $\mathrm{AC} \mathrm{Her} \mathrm{using} \mathrm{N}$ and Q-band imaging. Close et al. (2003) however detect no significant extended structure around AC Her, using adaptive optics at mid-infrared wavelengths with a higher spatial resolution.

RU Cen also is an RV Tauri star of spectroscopic class B and photometric class a. It is a regular pulsator with a pulsation period of 64.6 days, a mean magnitude of $m_{V}=9.05 \mathrm{mag}$ and an amplitude of $\Delta m_{V}=1.28 \mathrm{mag}$ (Pollard et al. 1996). During every formal pulsation cycle (the period between two photometric deep minima) two shock waves propagate through the atmosphere (Maas et al. 2002). The same paper reports the detection of a significant radial velocity variation on a longer time scale and attributes this to be due to orbital motion. No orbit could be determined, however.

Both stars are very regular RV Tauri pulsators with very similar chemical depletion patterns, atmospheric parameters and pulsational stability. In this paper we report our detailed comparative study of both objects based on our optical monitoring and Spitzer Space Telescope spectra. The outline of the paper is as follows: in Sect. 2 we give an overview of the different observations and reduction strategies. Section 3 contains the construction of the spectral energy distributions and colour excess determination. In Sect. 4 we discuss the spectral monitoring and deduce the binary model of RU Cen. The analysis of the infrared spectra and the spectral fitting is done in Sect. 5. In Sect. 6 we model the observed SEDs using a passive disc model. The discussion of our different results and our conclusions are presented in Sects. 7 and 8.

\section{Observations and reduction}

\subsection{Spitzer}

We used the Infrared Spectrograph (IRS) aboard the Spitzer Space Telescope in February 2005 to obtain high- and low-resolution spectra of RUCen. The spectra were observed with combinations of the short-low (SL), short-high (SH), longlow (LL) and long-high (LH) modules. SL $(\lambda=5.3-14.5 \mu \mathrm{m})$ and $\operatorname{LL}(\lambda=14.2-40.0 \mu \mathrm{m})$ spectra have a resolving power of $R=\lambda / \Delta \lambda \sim 100$ and the SH $(\lambda=10.0-19.5 \mu \mathrm{m})$ and $\mathrm{LH}$ $(\lambda=19.3-37.0 \mu \mathrm{m})$ spectra have a resolving power of $\sim 600$.

The spectra were extracted from the SSC raw data pipeline version S13.2.0 products, using the $\mathrm{c} 2 \mathrm{~d}$ Interactive Analysis reduction software package (Kessler-Silacci et al. 2006; Lahuis et al. 2006). This data processing includes bad-pixel correction, extraction, defringing and order matching. Individual orders are corrected for offsets, if necessary, by small scaling corrections to match the bluer order.

\section{2. $T I M M I 2$}

We completed our infrared spectra of RU Cen with spectra in the $10 \mu \mathrm{m}$ region, taken with the TIMMI 2 instrument mounted on the $3.6 \mathrm{~m}$ telescope at the ESO La Silla Observatory in March 2004. The low-resolution $(R \sim 160) \mathrm{N}$ band grism was used in combination with a 1.2 arcsec slit, the pixel scale in the spectroscopic mode of TIMMI 2 is 0.45 arcsec. For the reduction of the spectra we used the method described in van Boekel et al. (2005).

\subsection{CORALIE}

We extended the radial velocity monitoring reported by Maas et al. (2002) with new data obtained with the same spectrograph CORALIE attached to the same $1.2 \mathrm{~m}$ Swiss Euler telescope. In total we accumulated 151 raw spectra between June 2000 and July 2006 with a typical sampling of 3 runs of 10 days spread over every semester. The radial velocity was determined by offline cross-correlation using a spectral mask tuned to the spectral properties of RUCen (Maas et al. 2002). The internal error for every measurement was quantified by the standard deviation of a 50-point bisector through the cross-correlation profile. The bisector was determined on an equidistant sampling, starting from 2 times the width $(\sigma)$ of the Gaussian fit through the crosscorrelation profile down to the minimum. From January 2005 onwards, we used the HARPS-software release to determine the cross-correlation profile. We extended the baseline by including the radial velocity data of Pollard et al. (1997) which are based on high-quality high-resolution spectra.

\section{SED determination}

We collected photometric data so as to construct the spectral energy distributions (SEDs) homogeneously (De Ruyter et al. $2005,2006)$. The main problem in constructing such SEDs of pulsating stars with large amplitudes and often strong cycle-tocycle variability is that equally phased data are necessary. Since we do not have these data available, photometric maxima were used for the SED construction.

Our SED construction gives $E(B-V)=0.4 \pm 0.3$ for RU Cen and $E(B-V)=0.2_{-0.2}^{+0.3}$ for AC Her. The colour excess $E(B-V)$ determination method was adopted from De Ruyter et al. (2006). We estimate total extinction by dereddening observed photometry, using the average extinction law of Savage \& Mathis (1979). Minimising the difference between the dereddened observed fluxes and the adopted Kurucz model gives total colour excess $E(B-V)$. Model parameters for our programme stars (Table 1) are taken from Maas et al. (2002) and Van Winckel et al. (1998). 


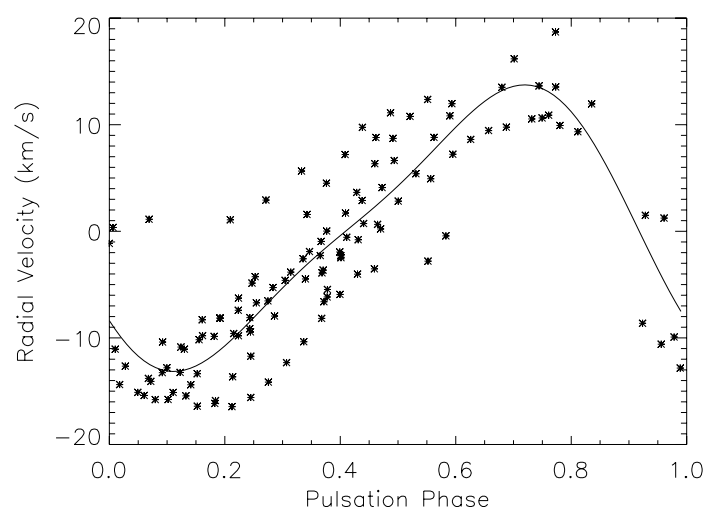

Fig. 1. The pulsational modulation of the radial velocity of RU Cen after cleaning of the orbit. The full line is the harmonic fit (one overtone included) with the pulsation period of 32.36 days. Zero phase was taken arbitrarily on the first datapoint obtained (JD 2448 388.93). The number of pulsation cycles covered is 170 . The fractional variance reduction of the fit is $77 \%$. Note the very high pulsational amplitude.

Table 1. The name, equatorial coordinates $\alpha$ and $\delta$ (J2000), the effective temperature $T_{\text {eff }}$, the surface gravity $\log g$ and the metallicity $[\mathrm{Fe} / \mathrm{H}]$ of the programme stars RU Cen and AC Her. Model parameters are taken from Maas et al. (2002) and Van Winckel et al. (1998). Typical errors are $\Delta T=250 \mathrm{~K}, \Delta \log g=0.5$.

\begin{tabular}{cccccc}
\hline \hline Name & $\begin{array}{c}\alpha(\mathrm{J} 2000) \\
(\mathrm{h} \mathrm{m} \mathrm{s})\end{array}$ & $\begin{array}{c}\delta(\mathrm{J} 2000) \\
\left({ }^{\circ}{ }^{\prime \prime}\right)\end{array}$ & $\begin{array}{c}T_{\text {eff }} \\
(\mathrm{K})\end{array}$ & $\begin{array}{c}\log g \\
(\mathrm{cgs})\end{array}$ & {$[\mathrm{Fe} / \mathrm{H}]$} \\
\hline RU Cen & 120923.7 & -452535 & 6000 & 1.5 & -2.0 \\
AC Her & 183016.2 & +215200 & 5500 & 0.5 & -1.5 \\
\hline
\end{tabular}

The SEDs of RUCen and ACHer have also been discussed in Maas et al. (2002) and De Ruyter et al. (2006) who find a total reddening for RU Cen of respectively $E(B-V)=0.6 \pm 0.1$ and $E(B-V)=0.3 \pm 0.3$ and a total reddening for AC Her of $E(B-V)=0.1_{-0.2}^{+0.3}$.

For both stars we find a broad infrared excess, starting around L-M. We find a value for the energy ratio $L_{\mathrm{IR}} / L_{*} \approx 0.15$ for RU Cen and $L_{\mathrm{IR}} / L_{*} \approx 0.35$ for AC Her.

\section{Binary orbit of RU Cen}

Together with ACHer, RUCen is known to be one of the most regular RV Tauri star pulsators. It has a stable formal period of 64.60 days and a total peak-to-peak amplitude of 1.3 mag in $V$ (Pollard et al. 1997). There is no long-term photometric modulation detected.

The pulsational modulation of the radial velocity is very significant (see Fig. 1) making orbital detection far from straightforward. Any cycle-to-cycle variability will make that a systematic cleaning of the pulsation from the raw radial velocity data yields a residual. Moreover, strong atmospheric shocks associated with the RV Tauri pulsations passing through the line-forming region (e.g. Gillet et al. 1990), have a strong effect on the line-profiles. This makes the determination of the stellar radial velocity at those pulsational phases problematic. In Fig. 2 we show a few cross-correlation profiles at different phases in the pulsation cycle. The propagation of the shock is well illustrated and in the case of RU Cen the non-linear behaviour leads to a very significant drop in velocity of more than $20 \mathrm{~km} \mathrm{~s}^{-1}$ over a small phase interval. The shocks in RU Cen are so energetic that during these phases, He lines are observed in emission (Maas et al. 2002).

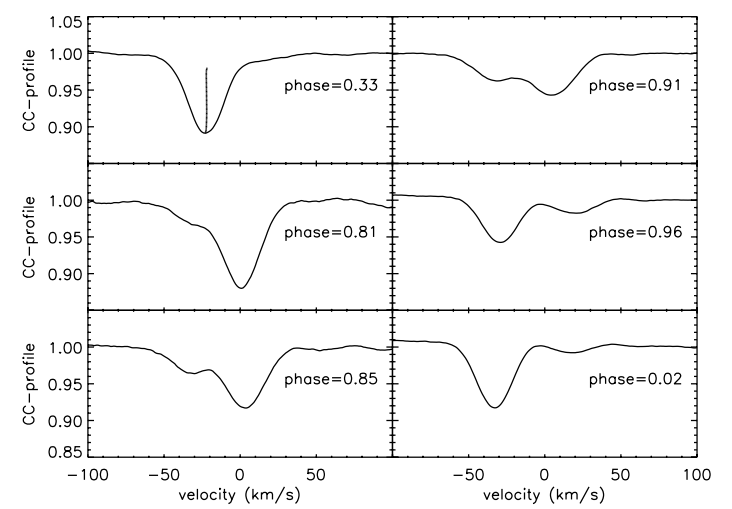

Fig. 2. The cross-correlation profiles obtained at different pulsational phases. The upper left panel gives the cross correlation profile at phase 0.33 with the 50 -point bisector which is used to quantify the radial velocity. In the other panels, we witness the propagation of a strong shock in the lineforming region of the atmosphere.

Table 2. The orbital elements of the binary RUCen. $P$ is the orbital period, JD the periastron passage, $K$ gives the semi-amplitude, $e$ the eccentricity, $\omega$ is the longitude of periastron, $\gamma$ is the system radial velocity, $a_{1}$ gives the semi-major axis of the primary and $i$ is the inclination.

\begin{tabular}{cccc}
\hline \hline Element & Value & Formal Error $(1 \sigma)$ & Unit \\
\hline$P$ & 1489 & 4 & days \\
JD $_{\text {periastron }}$ & 2451378 & 10 & days \\
$K$ & 21.9 & 0.7 & $\mathrm{~km} \mathrm{~s}^{-1}$ \\
$e$ & 0.60 & 0.03 & \\
$\omega$ & 133 & 4 & degrees \\
$\gamma$ & -28.4 & 0.4 & $\mathrm{~km} \mathrm{~s}^{-1}$ \\
rms & 4.5 & & $\mathrm{~km} \mathrm{~s}^{-1}$ \\
Mass Function & 0.83 & & $M_{\odot}$ \\
$a_{1} \sin i$ & 2.4 & & $\mathrm{AU}$ \\
\hline
\end{tabular}

Despite the strong pulsational modulation in the radial velocity data, variability in the radial velocity is detected with a much longer time scale. We interpret this as being due to orbital motion and modelled this with a Keplerian model of a binary star.

To obtain the orbital elements, we only retained the high quality data outside the pulsation phases where the strong shock is visible in the cross-correlation profile. To do so, we required that in all data used for the orbital detection, the 50-point bisector has a variance of less than $1 \mathrm{~km} \mathrm{~s}^{-1}$. We performed an iterative process on the raw velocity data in which we cleaned the orbital solution from the raw radial velocity data to obtain a good model of the pulsation cycle itself. We used PDM (Phase Dispersion Minimalisation method developed by Stellingwerf 1978) to quantify the fundamental pulsation period (time between successive deep and shallow photometric minimum) and determined a harmonic fit with one overtone as a model description of the pulsation. We then cleaned the raw radial velocity data by the pulsation model and performed the next least-square fit of the orbit. We stopped the iteration when the changes in the orbital parameters became less than the error.

The final result is that we indeed found an orbital solution with a period of $1489 \pm 4$ days. The errors given in the table are the formal errors obtained using the covariance matrix (Hadrava 2004). The mass function is $0.83 M_{\odot}$ and the semi-major axis is $a_{1} \sin i=2.4 \mathrm{AU}$. 


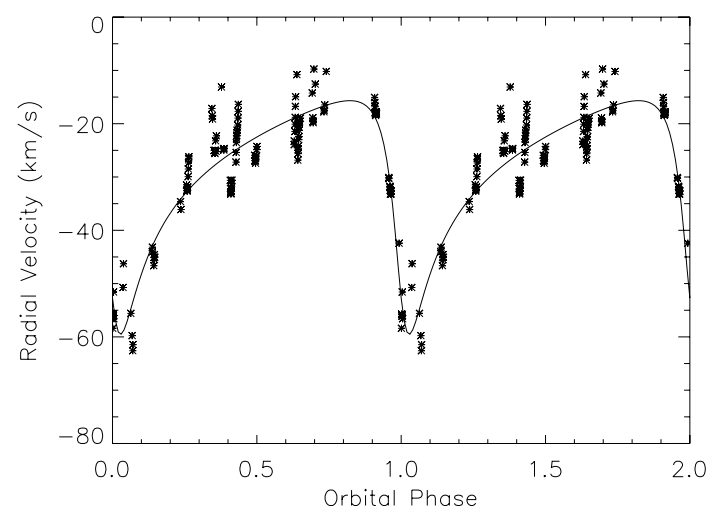

Fig. 3. The radial velocity data of RU Cen, cleaned for the pulsational modulation and folded on the orbital period of 1489 days. The epoch of zero phase is the periastron passage (JD 2451378). The full line represents the least square orbital solution discussed in the text. Note that for clarity the figure samples all data twice.

\section{Analysis infrared spectra}

\subsection{General}

A first look at the infrared spectra of AC Her and RU Cen shows their striking similarity (see Fig. 4), both in the global shape and in the dust emission features. In both spectra there is a lack of a strong $10 \mu \mathrm{m}$ amorphous silicate feature, while the $20 \mu \mathrm{m}$ amorphous feature is prominent. ACHer and RUCen show strong emission features around 11.3-16.2-19.7-23.7-28-33.6 $\mu \mathrm{m}$ which we can identify as features of forsterite and enstatite, two abundant silicate crystals. In none of the spectra is there evidence for a carbon-rich component. Not only are the infrared spectra of AC Her and RU Cen very similar to each other, they also show a strong resemblance to the infrared spectrum of the solar system comet Hale-Bopp (Bouwman et al. 2003; Min et al. 2005b).

Min et al. (2005b) model the thermal emission and degree of linear polarisation of radiation scattered by grains in the coma of the comet Hale-Bopp. The largest contribution in dust, about $75 \%$ of total dust mass, is made up of amorphous silicate grains, with dust sizes from $0.01 \mu \mathrm{m}$ up to $93 \mu \mathrm{m}$, and large amorphous carbon grains $(\sim 10 \mu \mathrm{m})$. Small crystalline silicates make up only $5 \%$ of the total dust mass of Hale-Bopp but this is sufficient to have this strong spectral signature in the IR spectrum.

\subsection{Feature identification}

The amorphous and crystalline features seen in the spectra of ACHer and RUCen are identified as caused by the most commonly found dust species in the circumstellar environment (Molster et al. 2002a,b,c; Min et al. 2007), namely glassy and crystalline silicates with an olivine and pyroxene stoichiometry. We further take the commonly used term "amorphous and crystalline olivine and pyroxene" to describe these dust species. Amorphous olivine $\left(\mathrm{Mg}_{2 x} \mathrm{Fe}_{2(1-x)} \mathrm{SiO}_{4}\right.$, where $0 \leq x \leq 1$ denotes the magnesium content) has very prominent broad features around $9.8 \mu \mathrm{m}$ and $18 \mu \mathrm{m}$. These features (also called the $10 \mu \mathrm{m}$ and $20 \mu \mathrm{m}$ features) arise respectively from the Si-O stretching mode and the O-Si-O bending mode. For large grains the $9.8 \mu \mathrm{m}$ feature gets broader and shifts to redder wavelengths. Amorphous pyroxene $\left(\mathrm{Mg}_{x} \mathrm{Fe}_{1-x} \mathrm{SiO}_{3}\right)$ shows a $10 \mu \mathrm{m}$ feature similar to that of amorphous olivine, but shifted towards shorter wavelengths. Also the shape of the $20 \mu \mathrm{m}$ feature is slightly different. Crystalline olivine and pyroxene have very distinct emission features and comparing with the features seen in the spectra

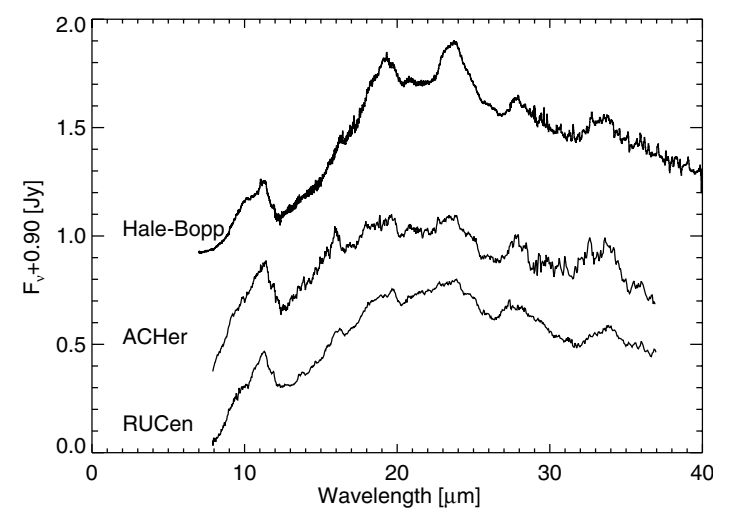

Fig. 4. ISO-SWS spectra from the solar system comet Hale-Bopp (Crovisier et al. 1997), the RV Tauri star AC Her (Molster et al. 1999) and combined TIMMI 2 and Spitzer-IRS spectrum of RU Cen. The spectra are normalised and offset for comparison. The dust emission features on top of the continuum are identified as being due to enstatite and forsterite grains.

of AC Her and RU Cen, we conclude that the Mg-rich end members, forsterite $\left(\mathrm{Mg}_{2} \mathrm{SiO}_{4}\right)$ and enstatite $\left(\mathrm{MgSiO}_{3}\right)$, dominate our spectra. Forsterite condenses directly from the gas-phase at high temperatures $(\approx 1500 \mathrm{~K})$ or it may form by thermal annealing of amorphous silicates, diffusing the iron out of the latticestructure. Enstatite can form in the gas-phase from a reaction between forsterite and silica, or it may also form by a similar thermal annealing process as forsterite (Bradley et al. 1983; Tielens et al. 1997).

The observed spectra of ACHer and RU Cen show a shift from the amorphous $18 \mu \mathrm{m}$ feature towards $20 \mu \mathrm{m}$ when comparing with synthetic spectra of amorphous olivine and pyroxene. This could point to the dominance of Mg-rich amorphous dust, which also shows this shift to redder wavelengths. Photospheric depletion in iron, which we detect in RU Cen and AC Her (Maas et al. 2002; Van Winckel et al. 1998), can be understood when the iron is locked up in the circumstellar dust (Waters et al. 1992). The lack of iron in the detected silicates is therefore surprising. If both the crystalline and amorphous silicates are devoid of iron, this could mean that iron is stored in metallic iron or iron-oxide (Sofia et al. 2006). Metallic iron has no distinct features but still a significant contribution in opacity, especially at shorter wavelengths, making it very hard to detect directly.

\subsection{Profile fitting}

Our aim is to fit the observed crystalline emission features of ACHer and RUCen with synthetic spectra of forsterite and enstatite. The conversion from laboratory measured optical constants of dust to mass absorption coefficients is not straightforward and is largely dependent on the adopted size, shape, structure and chemical composition of the dust (Min et al. $2003,2005 a)$. These different dust approximations result in very different emission feature profiles. The spectrum produced by homogeneous spherical particles is very different from that produced by more irregular particles. This difference is much larger than the difference between synthetic spectra computed using approximations of different irregular particles (Min et al. 2003). We have access to a large sample of mass absorption coefficients of various dust shapes and sizes. The sample consists of forsterite and enstatite in Mie approximation (Aden \& Kerker 1951; Toon \& Ackerman 1981), CDE (continuous distribution of ellipsoids, Bohren et al. 1983), GRF (Gaussian random field 


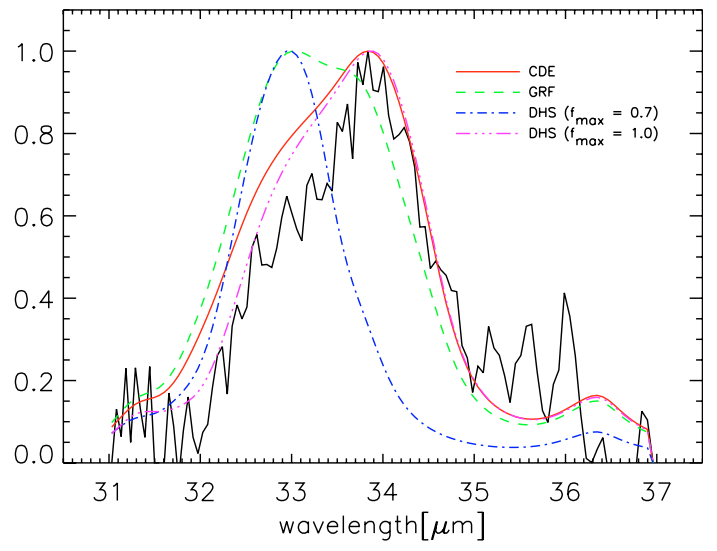

Fig. 5. Normalised and continuum subtracted $33.6 \mu \mathrm{m}$ emission feature of RU Cen together with mass absorption coefficients for different forsterite shape distributions. The DHS forsterite grains both have grain sizes of $0.1 \mu \mathrm{m}$. From the figure it is clear that the grains in DHS approximation must have $f_{\max }=1.0$. For this emission feature the difference between DHS grains of $0.1 \mu \mathrm{m}$ and $1.5 \mu \mathrm{m}$ is minimal.

particles, e.g. Grynko \& Shkuratov 2003; Shkuratov \& Grynko 2005) and DHS (distribution of hollow spheres, Min et al. 2003, 2005a) grain shapes. The DHS shaped particles are further characterised by the fraction of the total volume occupied by the central vacuum inclusion, $f$, over the range $0<f<f_{\max }$. The value of $f_{\max }$ reflects the degree of irregularity of the particles (Min et al. 2003, 2005a). Mie theory is used to model homogeneous spherical compact grains, while CDE, GRF and DHS particles are more irregular. Cross sections in CDE and GRF are computed under the assumption that the grains are in the Rayleigh limit (that the grains are much smaller than the wavelength of radiation, thus smaller than $0.1 \mu \mathrm{m}$ ). The different grain sizes for Mie and DHS dust particles range from $0.1 \mu \mathrm{m}$ till $10 \mu \mathrm{m}$. These different grain sizes produce emission features at very different central wavelengths (see Figs. 5 and 6) and larger grains mainly contribute to the dust continuum.

To keep the number of free parameters in our $\chi^{2}$ minimisation reasonable, we first determined and then fixed the best forsterite dust opacity description. We carefully compared the observed dust emission profiles of RUCen and ACHer with emission profiles of different adopted forsterite shapes. From Figs. 5 and 6 it is clear that different dust shapes result in very different emission profiles. We calculated that the best fit is obtained when using small $(<0.1 \mu \mathrm{m})$ forsterite particles in CDE approximation and big $(1.5 \mu \mathrm{m})$ forsterite particles in DHS approximation with $f_{\max }=1.0$. The identification of the best enstatite dust species is not as straightforward since the enstatite emission features are often blended with more prominent forsterite features. We have opted to keep the same dust size distribution for forsterite as for enstatite since this is physically more plausible.

\subsection{Full feature fitting}

Identifying the underlying dust continuum distribution is by no means straightforward. We opted to extract the dust continuum by connecting the local minima of the crystalline features. This method was used both for the synthetic spectra of forsterite and enstatite and the observed spectra of RUCen and AC Her to allow for a quantative comparison. The best model fit was then determined using a $\chi^{2}$ minimalisation. Free parameters are dust species, the fraction of the given dust species, dust temperatures

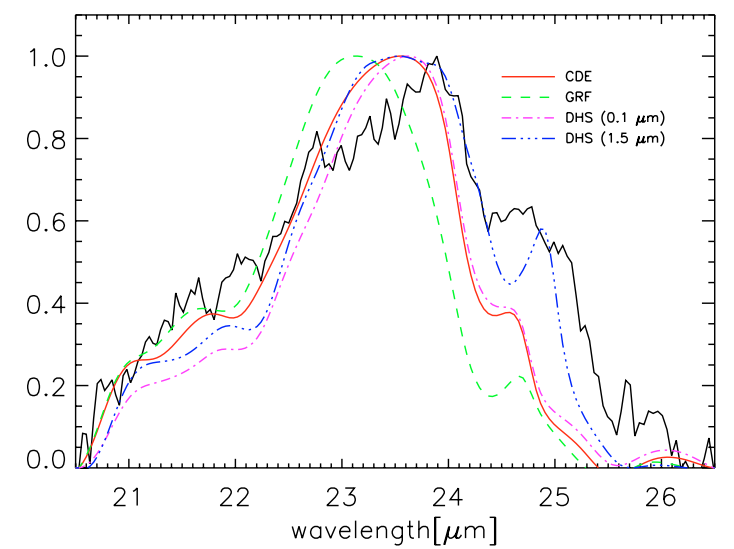

Fig. 6. Normalised and continuum subtracted $23.7 \mu \mathrm{m}$ emission feature of RU Cen together with mass absorption coefficients for different forsterite shape distributions. The DHS forsterite grains both have $f_{\max }=1.0$. Grains of $1.5 \mu \mathrm{m}$ are necessary to fit the observed bump at $25 \mu \mathrm{m}$.

and the dust fractions at a given dust temperature. This allows us to study the contribution of different dust temperatures and the different dust species to the infrared spectral features.

The model emission profiles are then given by

$F_{\lambda} \sim\left(\sum_{i} \alpha_{i} \kappa_{i}\right) *\left(\sum_{j} \beta_{j} B_{\lambda}\left(T_{j}\right)\right)$

where $\kappa_{i}$ is the mass absorption coefficient of dust component $i$ and $\alpha_{i}$ gives the fraction of that dust component, $B_{\lambda}\left(T_{j}\right)$ denotes the Planck function at temperature $T_{j}$ and $\beta_{j}$ the fraction of dust in that given temperature. In this approach we assume that different grain species and grain sizes can have equal temperatures and that the observed flux originates from an optically thin region.

From Sect. 5.3 we find that the best fit is obtained using very irregular grains, which was also found in recent studies of dust in comets and protoplanetary discs (e.g. Crovisier et al. 1997; Bouwman et al. 2001, 2003). As a next step, the dust species are kept fixed and the free parameters in the $\chi^{2}$ minimalisation are dust temperatures, temperature fractions and dust species fractions.

It is clearly not possible to fit the observed crystalline dust spectral features of RU Cen and AC Her with only one dust temperature (Fig. 7). The range of temperatures needed is, however, limited and good fits can be achieved by allowing only two different dust temperatures between $50 \mathrm{~K}$ and $1000 \mathrm{~K}$ for the adopted dust features (small/big forsterite/enstatite). An increase in the number of dust temperatures yielded only a minor improvement in the $\chi^{2}$ minimisation.

Results of our model fit are given in Table 3 and Figs. 8 and 9. We find that both hot and cool dust are necessary to reproduce the observed spectra and that AC Her and RU Cen have a similar temperature distribution. The hot dust temperature is less constrained and similar fits could be derived with a temperature a few hundreds of Kelvin higher. All our best models give a fraction of big enstatite grains that is higher than the fraction of small enstatite grains, sometimes the fraction of small grains 


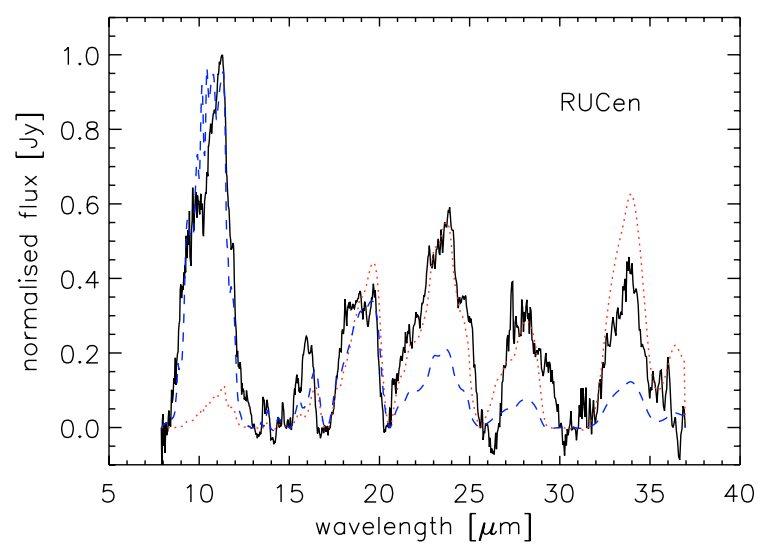

Fig. 7. Continuum subtracted and normalised spectrum of RUCen. Overplotted two models of a forsterite-enstatite mixture at different temperatures. The dashed line represents a model at $150 \mathrm{~K}$ and the dotted line a model at $600 \mathrm{~K}$. It is clear that a single-temperature model is insufficient to reproduce the spectrum of RU Cen.

Table 3. Dust parameters deduced from our spectral fitting for RU Cen and ACHer. Listed are the two dust temperatures, the fraction of dust with that given temperature and the different fractions of dust in the given dust species. Small forsterite/enstatite dust consists of CDE dust shapes with grain sizes $<0.1 \mu \mathrm{m}$, large forsterite/enstatite dust consists of DHS dust shapes of $1.5 \mu \mathrm{m}$ sized dust particles with $f_{\max }=1.0$.

\begin{tabular}{lcc}
\hline \hline & RUCen & AC Her \\
\hline$T_{1}=$ dust temperature 1 $(\mathrm{K})$ & 150 & 100 \\
$T_{2}=$ dust temperature 2 $(\mathrm{K})$ & 600 & 800 \\
$\beta_{1}=$ fraction dust in $T_{1}$ & 0.40 & 0.60 \\
$\beta_{2}=$ fraction dust in $T_{2}$ & 0.60 & 0.40 \\
$\alpha_{1}=$ fraction small forsterite & 0.40 & 0.50 \\
$\alpha_{2}=$ fraction large forsterite & 0.30 & 0.20 \\
$\alpha_{3}=$ fraction small enstatite & 0.00 & 0.10 \\
$\alpha_{4}=$ fraction large enstatite & 0.30 & 0.20 \\
\hline
\end{tabular}

is even zero. One explanation for the need of a high fraction of large grains is the rather broad $11.3 \mu \mathrm{m}$ feature, which can only be fitted using a considerable fraction of large grains. Including a contribution of amorphous silicates in this broad feature makes the crystalline emission feature much narrower and more peaked around $11.3 \mu \mathrm{m}$, which is distinctive for small forsterite grains. Of all the different observed emission features, the $11.3 \mu \mathrm{m}$ feature is most sensitive to adopted grain size. Molster et al. (2002b) already found for AC Her that the $11.3 \mu \mathrm{m}$ feature is well fitted by only crystalline silicates but that an amorphous contribution could not be excluded. Including an amorphous component would reduce the $11.3 \mu \mathrm{m}$ feature and result in a larger fraction of small forsterite grains and a reduction in big enstatite grains. The maximum dust temperature of the forsterite-enstatite mixture decreases from temperatures around $700 \mathrm{~K}$ to around $500 \mathrm{~K}$.

Our best models do not always match the observed features: in our modelled RU Cen spectrum the $19 \mu \mathrm{m}$ feature is stronger than the $23 \mu \mathrm{m}$ feature, while this is not the case in the observed spectrum of RU Cen. This problem could arise from a problem in the data reduction, since at around $20 \mu \mathrm{m}$, getting a good order overlap of the different echelle orders proved to be problematic. Also the shape of the $27 \mu \mathrm{m}$ feature is quite different in observed and modelled spectra. Another puzzling fact is that the $16.2 \mu \mathrm{m}$ forsterite feature seems to be shifted to the left and it is surprisingly strong in ACHer. This is not a temperature effect and it may call for the inclusion of another mineral since in RUCen this feature is not well reproduced either.

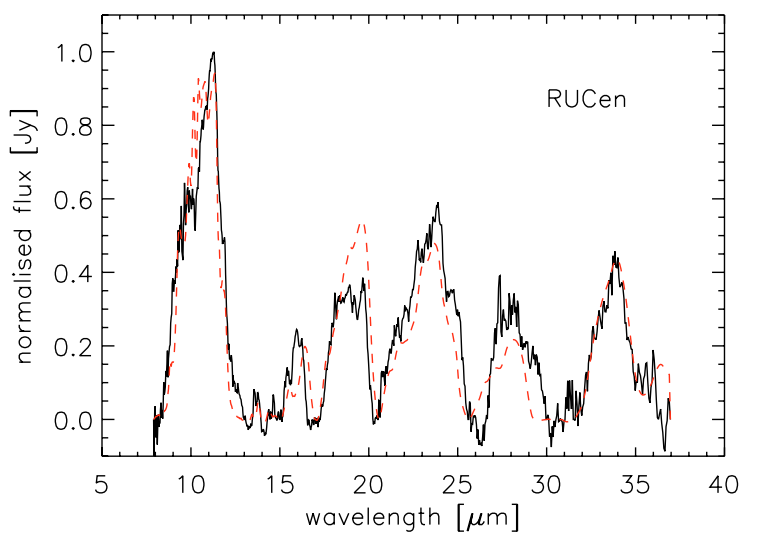

Fig. 8. Best model fit to the continuum subtracted and normalised spectrum of RU Cen. Fit parameters are given in Table 3.

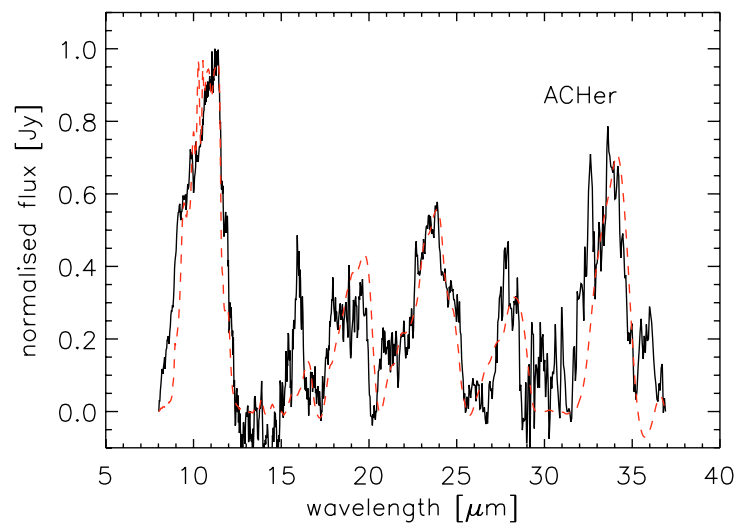

Fig. 9. Best model fit to the continuum subtracted and normalised spectrum of AC Her. Fit parameters are given in Table 3.

\section{SED fitting disc model}

\subsection{Method}

Broad-band SEDs are notoriously degenerate but together with the Spitzer infrared spectral information, the circumstellar physical characteristics are much better constrained. A firm first conclusion is that all spherical models failed to fit both the SED and the infrared spectral data. Moreover, spherical outflow models which fit the SED have evolutionary timescales which are much too short compared to any evolutionary track in which a postAGB star of spectral type F is involved. We therefore concentrate on constructing detailed 2D disc models.

\subsubsection{D disc model}

As a next step we performed an SED-fitting using a Monte Carlo code, assuming 2D-radiative transfer in a passive disc model (Dullemond et al. 2001, 2003; Dullemond \& Dominik 2004). This code computes the temperature structure and density of the disc. The vertical scale height of the disc is computed by an iteration process, demanding vertical hydrostatic equilibrium. The dust grain property distribution is fully homogeneous, and although this model can reproduce the SED, dust settling timescales indicate that settling of large grains to the midplane occurs and thus that an inhomogeneous disc model is necessary. Large grains are necessary to account for the $850 \mu \mathrm{m}$ flux in ACHer. As we do not possess a $850 \mu \mathrm{m}$ fluxpoint for RUCen, we estimated the $850 \mu \mathrm{m}$ flux by assuming a blackbody slope from the IRAS $60 \mu \mathrm{m}$ flux redwards, as is observed 
in ACHer. This submillimeter data is invaluable to constrain grain sizes in the disc. In all other similar sources sampled (De Ruyter et al. 2006) the $850 \mu \mathrm{m}$ flux shows that the flux is at the Rayleigh-Jeans slope connecting the $60 \mu \mathrm{m}$ IRAS flux point.

Dust settling time for a grain to migrate from height $z_{0}$ to $z$ is calculated using

$t_{\mathrm{set}}=\frac{\pi}{2} \frac{\Sigma_{0}}{\rho_{\mathrm{d}} a} \frac{1}{\Omega_{k}} \ln \frac{z}{z_{0}}$

with $\Sigma_{0}$ the surface density, $\rho_{\mathrm{d}}$ the particle density, $a$ the grain size and $\Omega_{k}=\sqrt{\frac{G M_{*}}{r^{3}}}$ the Kepler rotation rate (Miyake \& Nakagawa 1995). From our SED modelling (Sect. 6.2) we find that the surface density of these discs approximately is $\Sigma=$ $\Sigma_{0}\left(\frac{R}{1 \mathrm{AU}}\right)^{-0.5}$, with $\Sigma_{0} \approx 0.2 \mathrm{~g} \mathrm{~cm}^{-2}$. This estimation means that grains larger than $100 \mu \mathrm{m}$ can descend a distance of $50 \mathrm{AU}$ in less than $4 \times 10^{5}$ years. Grains of $850 \mu \mathrm{m}$ will even travel this distance in less than $6 \times 10^{4}$ years. This is similar to the estimated lifetime (Sect. 1) of the disc and means that there will be a vertical distribution of grain size where the largest grains settle in the disc midplane.

The disc structure and mid-IR flux is almost fully determined by the small grains while the cool midplane, consisting of large grains, mainly contributes to the long wavelength part of the SED and the total mass of the disc. We construct an inhomogeneous 2-component model where the near- and mid-IR flux comes from the small grains to which we add a blackbody flux to represent the midplane.

Stellar input parameters of the model are luminosity, mass (which we take fixed at $L=3000 L_{\odot}$ and $M=1 M_{\odot}$ ) and $T_{\text {eff }}$. Input disc parameters are $R_{\text {out }}$ and $R_{\text {in }}$, where the dust sublimation radius is used as a zero-order approximation of $R_{\text {in }}$, the total disc mass and the powerlaw for the density distribution. For the dust sublimation temperature for silicates we use the typical value $T_{\text {sub }}=1500 \mathrm{~K}$ and we assume blackbody radiation. For RU Cen and AC Her this gives $R_{\text {sub }} \approx 2 \mathrm{AU}$. In the more detailed modelling we take the inner radius of the disc so that the start of the IR-excess fits the photometric data. Since we are not dealing with outflow sources a powerlaw $>-2$ is used. Using this disc model, the SED can now be calculated, given a specific inclination angle of the system.

\subsection{Results}

The SED-fitting gives an estimate of the distance to the systems, $d=2.4 \mathrm{kpc}$ for RUCen and $d=1.4 \mathrm{kpc}$ for AC Her, using a luminosity $L=3000 L_{\odot}$. This distance estimation is largely dependent on luminosity of the star and adopted inclination of the system. Independent distances estimated from the P-L relation of Alcock et al. (1998) are $d=1.6 \pm 0.6 \mathrm{kpc}$ for RU Cen and $d=1.3 \pm 0.4 \mathrm{kpc}$ for AC Her (De Ruyter et al. 2006). For RU Cen this could mean that the adopted luminosity of $L=3000 L_{\odot}$ is too high.

When modelling the near- and mid-IR part of the SED the feature-to-continuum ratio of the silicate features is too strong in comparison with the ratio observed in the infrared spectra. Including an extra continuum opacity source is needed to reduce the strength of the features. Since the silicates are devoid of iron (Sect. 5.2), metallic iron is a potential opacity source: while its near-IR opacity is large, the absorption coefficient is unfortunately featureless so direct detection is difficult. Inclusion of metallic iron has a strong impact on the modelling because the near-IR excess increases significantly with a given inner radius. The opacity of metallic iron alone would require an inner radius

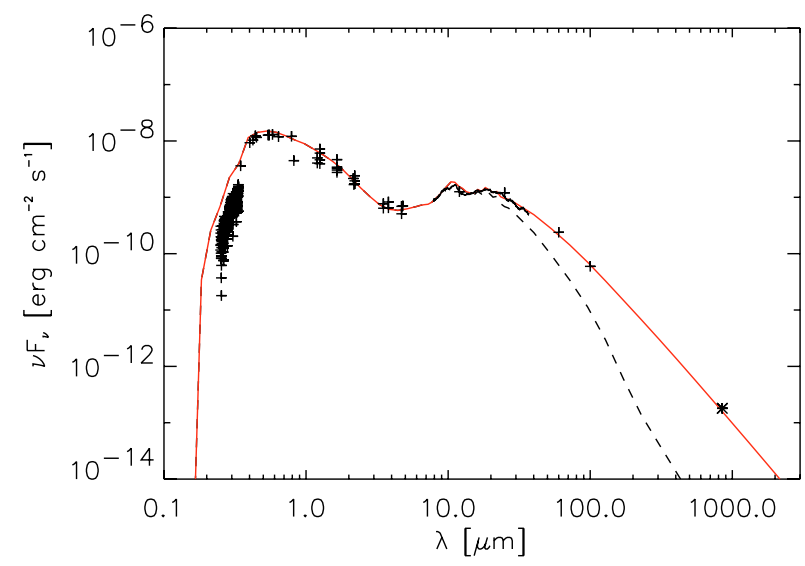

Fig. 10. SED disc modelling of RUCen. The dashed line represents the homogeneous disc model consisting of grains between $0.1 \mu \mathrm{m}$ and $20 \mu \mathrm{m}$. The red line gives the disc model with an added blackbody to represent the cool midplane. Crosses represent photometric data and the solid black line the observed Spitzer spectrum. Note that the $850 \mu \mathrm{m}$ photometric point (asterisk) is an estimation.

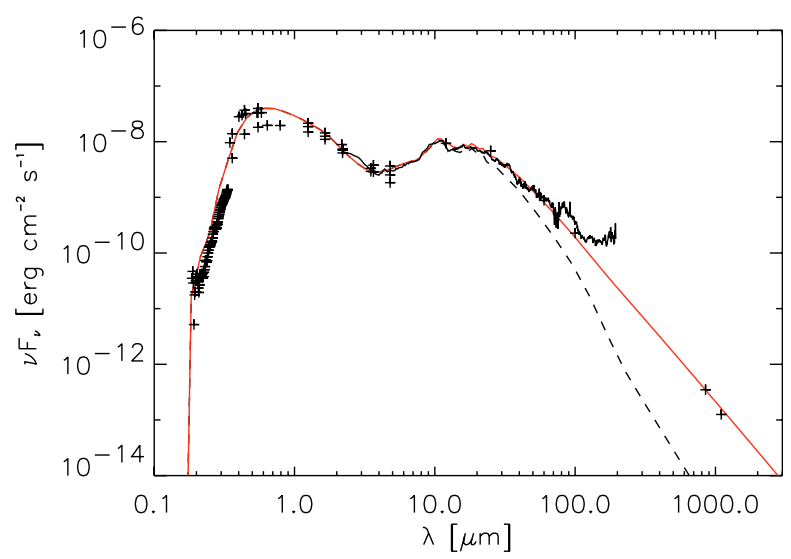

Fig. 11. SED disc modelling of ACHer. The dashed line represents the homogeneous disc model consisting of grains between $0.1 \mu \mathrm{m}$ and $20 \mu \mathrm{m}$. The red line gives the disc model with an added blackbody to represent the cool midplane. Crosses represent photometric data and the solid black line the observed ISO-SWS spectrum.

$>70$ AU to maintain a reasonable fit to the SED, but this is inconsistent with the constraints from high spatial resolution imaging Close et al. (2003). So relatively large grains (up to $20 \mu \mathrm{m}$ ) were included in the modelling as well.

Our final model of the near- and mid-infrared part of the SED with a homogeneous disc model consists of grains with sizes between $0.1 \mu \mathrm{m}$ and $20 \mu \mathrm{m}$. In a disc, grain evolution can produce larger grains than what is typically used for ISM grains. A grain size distribution $\sim a^{p}$ ( $a$ is the grain size) with a powerlaw $3.5<p<-2.5$ is expected (D'Alessio et al. 2001). Bouwman et al. (2003) for example find for the Herbig Be star HD 100546, a grain size distribution $p \sim-2$ and $p \sim-2.8$ for the comet Hale-Bopp. We take $p=-3.0$ for the full range of grain sizes between 0.1 and $20 \mu \mathrm{m}$ to still have a significant component of small grains. Further disc input parameters are adopted dust species, for which we take a mixture of $6 \%$ metallic iron and $94 \%$ amorphous and crystalline silicates. Best models for the SED-fit of RU Cen and AC Her are presented in Figs. 10 and 11. For RU Cen we find a model with $R_{\text {in }}=35 \mathrm{AU}, R_{\text {out }}=400 \mathrm{AU}$ and a total disc mass (in small grains and gas) of $3 \times 10^{-4} M_{\odot}$. AC Her has a disc model with $R_{\text {in }}=35 \mathrm{AU}, R_{\text {out }}=300 \mathrm{AU}$ and a 


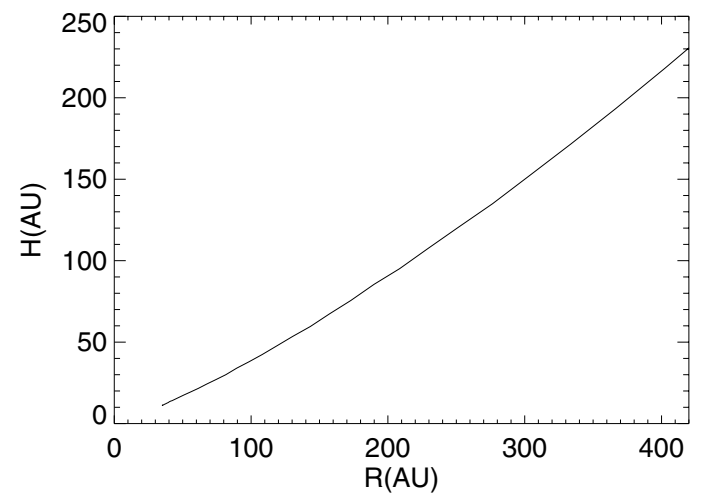

Fig. 12. The scaleheight - radius relation $H \approx 0.15 \times R^{1.2}$, found for the discs of RU Cen and AC Her.

total disc mass (in small grains and gas) of $5 \times 10^{-4} M_{\odot}$. For both stars we find that the best fit is obtained when using a rather flat surface density distribution $\left(\Sigma \sim R^{\alpha}\right.$, with $\left.\alpha>-1.0\right)$. To reduce the number of free parameters we kept the value of this powerlaw fixed at -0.5 . The inclusion of both metallic iron and larger grains cause a degeneracy in the value for the inner radius. If one would increase the fraction of large grains, as main contributors to the continuum opacity, the inner radius could have values between $15 \mathrm{AU}$ and $30 \mathrm{AU}$. This is still significantly larger than the sublimation radius for these stars (Sect. 6.1.1). The larger inner radius could be an evolutionary effect of the disc. Interferometric measurements are clearly needed to further constrain the disc radii. The added blackbody to represent the midplane and explain the far-IR part of the SED has a temperature of $120 \mathrm{~K}$ for RU Cen and $170 \mathrm{~K}$ for AC Her.

These temperatures and the $850 \mu \mathrm{m}$ fluxes can be used to estimate the dust mass in these large grains. In the optical thin approach (at $850 \mu \mathrm{m}$ ) the disc mass can be estimated by using (Hildebrand 1983)

$M_{\mathrm{d}}=\frac{F_{850} D^{2}}{\kappa_{850} B_{850}(T)}$.

Assuming a cross section of large spherical grains, the mass absorption coefficient $\kappa=\frac{\pi a^{2}}{\frac{4}{3} \pi a^{3} \rho}$, with $a$ the grain size and $\rho$ typically $3.3 \mathrm{~g} \mathrm{~cm}^{-3}$ for astronomical silicate, of $850 \mu \mathrm{m}$ grains in blackbody approximation is about $2.4 \mathrm{~cm}^{2} \mathrm{~g}^{-1}$. This results in dust mass estimates of $5 \times 10^{-4} M_{\odot}$ for RU Cen and $2 \times 10^{-4} M_{\odot}$ for AC Her.

The resulting discs for RUCen and AC Her are flared discs, with the scale height $H \approx 0.15 \times R^{1.2}$ (Fig. 12). This enables the discs to reprocess the large fraction, $15 \%$ for RUCen and $35 \%$ for AC Her (Sect. 3), of light emitted by the central star.

The models for RUCen and AC Her have comparable discs, with similar geometries, scale heights, temperature distributions and dust grain sizes. Temperature distributions in the discs are in agreement with dust temperatures derived from the spectral fitting. Both discs are optically thick in the equator direction between $0.1 \mu \mathrm{m}$ and $1 \mu \mathrm{m}$. After $1 \mu \mathrm{m}$ the optical thickness decreases rapidly, making the disc optically thin for infrared emission.

\section{Discussion}

A major problem in the SED model fitting of the the near- and mid-infrared flux is that the models are degenerate, especially the outer radius and the total disc mass are poorly constrained.
Almost equally fitting models for RUCen and ACHer can be found with an outer radius a few hundred AU larger which would result in a larger total disc mass. It is also difficult to discriminate between the two opacity sources we added to reduce the feature-to-continuum ratio of the silicate features: metallic iron and larger grains. If grains up to $50 \mu \mathrm{m}$ are included in the disc, the amount of metallic iron needed to fit the spectrum will be reduced significantly.

To compare the modelled SED with observational photometric data, these data need to be corrected for the interstellar extinction. A value for the interstellar extinction would constrain the inclination of the system. Since no such estimate is available we put a minimum on the interstellar extinction of $E(B-V)_{i s}=0.1$. As a maximum value we use the total extinction which we have deduced in Sect. 3. This gives us possible inclination values smaller than $70^{\circ}$ for RU Cen and smaller than $50^{\circ}$ for $\mathrm{AC} \mathrm{Her}$.

With these values for the inclination a minimal mass for the companion star can be estimated, using the mass function and a typical value for the primary of $M_{1}=0.5-0.6 M_{\odot}$. The mass functions of $f(M)=0.83 M_{\odot}$ for RUCen and $f(M)=$ $0.25 M_{\odot}$ for AC Her (Van Winckel et al. 1998) yield a minimal mass for the companion star of $M_{2}=1.7 M_{\odot}$ for RUCen and $M_{2}=1.1 M_{\odot}$ for AC Her. Even the minimal mass of the companion of RUCen is therefore not compliant with a possible white dwarf mass, and the companions are in both cases likely to be unevolved main sequence stars.

\section{Conclusion}

AC Her and RU Cen are known to be proto-typical RV Tauri pulsators which are normally seen as transition objects in their evolution from the AGB to the PNe evolutionary phase. Furthermore RU Cen turned out to be an evolved binary with an orbital period similar to AC Her indicating that it must have been subject to severe binary interaction when at giant dimensions. Neither of the two currently fills its Roche Lobe. In this paper we focused on modelling the circumstellar environment as constrained by our high quality Spitzer spectra and the broad-band SED. Our analysis showed that both stars are surrounded by a circumbinary dusty disc in hydrostatic equilibrium. Since the disc modelling is a degenerate problem, interferometric measurements are needed to constrain further the disc geometry (e.g. Deroo et al. 2007a,b).

The observed Spitzer spectra clearly show that the circumstellar grains are extremely processed. The IR-spectrum of both objects is dominated by crystalline dust features. The mineralogy is magnesium rich and large grains and/or metallic iron is necessary to explain the low feature-to-continuum ratio of the silicate features. The temperature estimate of the crystalline silicates show that a significant fraction must be rather cool, and certainly well below the annealing temperature. This shows that either radial mixing from the hot inner boundary (where annealing can take place) to far out in the disc must have occurred or that the formation process and thermal history of the grains is quite different in discs than in outflows. The profile fitting shows that the grains must be very irregular.

Despite the very different evolutionary history and the very different evolutionary timescales involved, it is remarkable that the mineralogy of the small hot silicates around the evolved objects RU Cen and AC Her is extremely similar to what is found in some young stellar objects (YSO) such as HD 100546 (Malfait et al. 1998; Lisse et al. 2007) and that of primitive comets such as Hale-Bopp (Bouwman et al. 2003; Lisse et al. 2007; Min et al. $2005 b)$. In all those systems, the thermal history of the grains has been such as to promote the dominance of forsterite, the 
Mg-rich end member of the crystalline olivine family. In YSO as well as in comets, the dust processing is thought to be the tracer for the process of disc clearing and planet building in the proto-planetary disc.

In evolved objects the circumstellar material is coming from the stars themselves and is much more chemically homogeneous than the ISM composition around YSO. Moreover the evolutionary timescales are likely to be orders of magnitude smaller than the disc evolution in YSO. Detailed dust mineralogy studies around evolved stars can therefore yield important information on dust formation and dust processing in discs, and this under very different chemical, dynamical and evolutionary environments than the processing in proto-planetary discs. Our study appears to indicate that the chemico-physical processes of dust grains in the hydrostatic discs of the evolved binaries RUCen and ACHer, are very similar to those governing in the protoplanetary discs around YSO. We are in the process of expanding our study to a wider sample of evolved binaries. This will enable us to build up a broader view of the chemico-physical dust processes of the grains around rapidly evolving stars.

The inclination and mass function give a minimal mass for the companion star of $1.1 M_{\odot}$ for ACHer and $1.7 M_{\odot}$ for RU Cen. The unevolved companions have masses such that they would normally (on single-star evolutionary tracks) evolve to carbon stars on the AGB. Given the silicate dominated circumstellar dust, it is however, clear that even the primaries did not evolve to become carbon stars. The actual orbits combined with the observed chemical evolution of the stars show that the binary interaction processes dominate the final evolution of these objects. The internal chemical evolution seems to have been cut short by binary interaction processes. As such the stars do not evolve on single-star evolutionary tracks and both objects should be seen as remarkably evolved binaries. It is likely that the formation of the circumstellar dust in these objects is closely related to binary interaction.

Both objects show that the formation, structure and evolution of the circumstellar discs play a leading role in their final evolution and illustrate once again the intimate relation between the depleted photospheres and the presence of a circumbinary disc. Since post-AGB stars with similar SEDs and/or chemistry are abundant (e.g. De Ruyter et al. 2006; Reyniers \& van Winckel 2007) it is clear that disc formation is a process relevant in the late evolution of a considerable fraction of binary stars.

Acknowledgements. The authors want to acknowledge: the Geneva Observatory and its staff for the generous time allocation on the Swiss Euler telescope; the $1.2 \mathrm{~m}$ Mercator staff as well as the observers from the Instituut voor Sterrenkunde who contributed to the monitoring observations using both the Euler and Mercator telescopes. C.G. acknowledges support of the Fund for Scientific Research of Flanders (FWO) under the grant G.0178.02. and G.0470.07. We also thank Fred Lahuis for his assistance with the Spitzer data reduction.

\section{References}

Aden, A. L., \& Kerker, M. 1951, J. Appl. Phys., 22, 1242

Alcock, C., Allsman, R. A., Alves, D. R., et al. 1998, AJ, 115, 1921

Blöcker, T. 1995, A\&A, 299, 755

Bohren, C. F., Huffman, D. R., \& Kam, Z. 1983, Nature, 306, 625

Bouwman, J., Meeus, G., de Koter, A., et al. 2001, A\&A, 375, 950

Bouwman, J., de Koter, A., Dominik, C., \& Waters, L. B. F. M. 2003, A\&A, 401, 577

Bradley, J. P., Brownlee, D. E., \& Veblen, D. R. 1983, Nature, 301, 473
Bujarrabal, V., Bachiller, R., Alcolea, J., \& Martin-Pintado, J. 1988, A\&A, 206, L17

Close, L. M., Biller, B., Hoffmann, W. F., et al. 2003, ApJ, 598, L35

Crovisier, J., Leech, K., Bockelee-Morvan, D., et al. 1997, Science, 275, 1904

D’Alessio, P., Calvet, N., \& Hartmann, L. 2001, ApJ, 553, 321

De Ruyter, S., van Winckel, H., Dominik, C., Waters, L. B. F. M., \& Dejonghe, H. 2005, A\&A, 435, 161

De Ruyter, S., van Winckel, H., Maas, T., et al. 2006, A\&A, 448, 641

Deroo, P., Acke, A., Verhoelst, T., et al. 2007a, A\&A, 468

Deroo, P., van Winckel, H., Verhoelst, T., et al. 2007b, A\&A, 467, 1093

Dullemond, C. P., \& Dominik, C. 2004, A\&A, 417, 159

Dullemond, C. P., Dominik, C., \& Natta, A. 2001, ApJ, 560, 957

Dullemond, C. P., van den Ancker, M. E., Acke, B., \& van Boekel, R. 2003, ApJ, 594, L47

Gillet, D., Duquennoy, A., Bouchet, P., \& Gouiffes, C. 1989, A\&A, 215, 316

Gillet, D., Burki, G., \& Duquennoy, A. 1990, A\&A, 237, 159

Giridhar, S., Rao, N. K., \& Lambert, D. L. 1994, ApJ, 437, 476

Giridhar, S., Lambert, D. L., \& Gonzalez, G. 1998, ApJ, 509, 366

Giridhar, S., Lambert, D. L., \& Gonzalez, G. 2000, ApJ, 531, 521

Gonzalez, G., Lambert, D. L., \& Giridhar, S. 1997a, ApJ, 481, 452

Gonzalez, G., Lambert, D. L., \& Giridhar, S. 1997b, ApJ, 479, 427

Grynko, Y., \& Shkuratov, Y. 2003, J. Quantitative Spectroscopy and Radiative Transfer, 78, 319

Hadrava, P. 2004, in Spectroscopically and Spatially Resolving the Components of the Close Binary Stars, ed. R. W. Hilditch, H. Hensberge, \& K. Pavlovski, ASP Conf. Ser., 318, 86

Hildebrand, R. H. 1983, QJRAS, 24, 267

Jura, M. 1986, ApJ, 309, 732

Jura, M., \& Kahane, C. 1999, ApJ, 521, 302

Jura, M., Balm, S. P., \& Kahane, C. 1995, ApJ, 453, 721

Jura, M., Chen, C., \& Werner, M. W. 2000, ApJ, 541, 264

Kessler-Silacci, J., Augereau, J.-C., Dullemond, C. P., et al. 2006, ApJ, 639, 275

Lahuis, F., et al. 2006, c2d Spectroscopy Explanatory Supplement, Cores to Disks, Spitzer Legace Team (Pasadena: Spitzer Science Center)

Lisse, C. M., Kraemer, K. E., Nuth, J. A., Li, A., \& Joswiak, D. 2007, Icarus, 187,69

Lloyd Evans, T. 1985, MNRAS, 217, 493

Maas, T., Van Winckel, H., \& Waelkens, C. 2002, A\&A, 386, 504

Maas, T., Van Winckel, H., \& Lloyd Evans, T. 2005, A\&A, 429, 297

Malfait, K., Waelkens, C., Waters, L. B. F. M., et al. 1998, A\&A, 332, L25

Min, M., Hovenier, J. W., \& de Koter, A. 2003, A\&A, 404, 35

Min, M., Hovenier, J. W., \& de Koter, A. 2005a, A\&A, 432, 909

Min, M., Hovenier, J. W., de Koter, A., Waters, L. B. F. M., \& Dominik, C. 2005b, Icarus, 179, 158

Min, M., Waters, L. B. F. M., de Koter, A., et al. 2007, A\&A, 462, 667

Miyake, K., \& Nakagawa, Y. 1995, ApJ, 441, 361

Molster, F. J., Yamamura, I., Waters, L. B. F. M., et al. 1999, Nature, 401, 563

Molster, F. J., Waters, L. B. F. M., \& Tielens, A. G. G. M. 2002a, A\&A, 382, 222

Molster, F. J., Waters, L. B. F. M., Tielens, A. G. G. M., \& Barlow, M. J. 2002b, A\&A, 382, 184

Molster, F. J., Waters, L. B. F. M., Tielens, A. G. G. M., Koike, C., \& Chihara, H. 2002c, A\&A, 382, 241

Pollard, K. R., Cottrell, P. L., Kilmartin, P. M., \& Gilmore, A. C. 1996, MNRAS, 279, 949

Pollard, K. R., Cottrell, P. L., Lawson, W. A., Albrow, M. D., \& Tobin, W. 1997, MNRAS, 286, 1

Preston, G. W., Krzeminski, W., Smak, J., \& Williams, J. A. 1963, ApJ, 137, 401

Reyniers, M., \& van Winckel, H. 2007, A\&A, 463, L1

Savage, B. D., \& Mathis, J. S. 1979, ARA\&A, 17, 73

Shenton, M., Evans, A., \& Williams, P. M. 1995, MNRAS, 273, 906

Shkuratov, Y. G., \& Grynko, Y. S. 2005, Icarus, 173, 16

Sofia, U. J., Gordon, K. D., Clayton, G. C., et al. 2006, ApJ, 636, 753

Stellingwerf, R. F. 1978, ApJ, 224, 953

Tielens, A. G. G. M., Waters, L. B. F. M., Molster, F. J., \& Justtanont, K. 1997, Ap\&SS, 255, 415

Toon, O. B., \& Ackerman, T. P. 1981, Appl. Opt., 20, 3657

van Boekel, R., Min, M., Waters, L. B. F. M., et al. 2005, A\&A, 437, 189

Van Winckel, H., Waelkens, C., \& Waters, L. B. F. M. 1995, A\&A, 293, L25

Van Winckel, H., Waelkens, C., Waters, L. B. F. M., et al. 1998, A\&A, 336, L17

Van Winckel, H., Waelkens, C., Fernie, J. D., \& Waters, L. B. F. M. 1999, A\&A, 343, 202

Waters, L. B. F. M., Trams, N. R., \& Waelkens, C. 1992, A\&A, 262, L37

Zsoldos, E. 1993, A\&A, 268, 149 